\title{
Expression level of epithelial cell adhesion molecule (EpCAM) of circulating tumor cells (CTCs) of patients with NSCLC as an early indicator to monitor the effects of postoperative adjuvant chemotherapy
}

\author{
De Zhi Zhen ${ }^{1}$, Yun Song Li ${ }^{2}$, Chong Yu Su${ }^{2}, \mathrm{Xu} \mathrm{Cheng}^{2}$, Shi Jie Zhou ${ }^{2}$, Yi Han ${ }^{2}$, Da Ping Yu², \\ Xiao Yun Song ${ }^{2}$, Ning $\mathrm{Xiao}^{2}$, Zhi Dong Liu ${ }^{2}$, Feng Wang \\ ${ }^{1}$ Department of Thoracic Surgery, Beijing TianTan Hospital, Capital Medical University, Beijing, China; ${ }^{2}$ Department of Thoracic Surgery, Beijing \\ Chest Hospital, Capital Medical University, Beijing, China; ${ }^{3}$ Department of Breast Surgery, Beijing TianTan Hospital, Capital Medical University, \\ Beijing, China \\ Contributions: (I) Conception and design: DZ Zhen, YS Li; (II) Administrative support: ZD Liu, F Wang; (III) Provision of study materials or \\ patients: CY Su, X Cheng, SJ Zhou, Y Han; (IV) Collection and assembly of data: All authors; (V) Data analysis and interpretation: DZ Zhen, CY Su, \\ X Cheng; (VI) Manuscript writing: All authors; (VII) Final approval of manuscript: All authors. \\ Correspondence to: Zhi Dong Liu, PhD. Department of Thoracic Surgery, Beijing Chest Hospital, Capital Medical University, No. 97 MaChang, \\ Tongzhou District, Beijing, 101149, China. Email: supermandream@aliyun.com; Feng Wang. Department of Breast Surgery, Beijing Tiantan \\ Hospital, Capital Medical University, No. 119, NanSiHuan West Road, Fengtai District, Beijing 100070, China. Email: 16911475@qq.com.
}

\begin{abstract}
Background: An early indicator for monitoring the effect of adjuvant treatment after lung cancer surgery is urgently needed. The study was to explore the effects of epithelial cell adhesion molecule (EpCAM) of circulating tumor cells (CTCs) in NSCLC patients with postoperative adjuvant chemotherapy.

Methods: Two drugs (platinum-containing chemotherapeutics + platinum-free chemotherapeutics) firstline chemotherapy regimen were given after surgery. MRNA of EpCAM was detected. Chest computed tomography, head computed tomography and abdominal B-ultrasound were reviewed before the first and third chemotherapy.
\end{abstract}

Results: EpCAM in CTCs from peripheral blood between the recurrent group and the non-recurrent group at 1 day before surgery, first, second and third adjuvant chemotherapy were no significant differences $(\mathrm{P}>0.05)$. Only one day before the fourth adjuvant chemotherapy treatment, it showed significant difference between the recurrent group and the non-recurrent group $(\mathrm{P}=0.008)$. There was a significant difference between the time of imaging diagnosis of recurrence or metastasis and the time of monitoring the expression level of EpCAM in CTCs from peripheral blood $(\mathrm{P}<0.0001)$.

Conclusions: EpCAM in CTCs from peripheral blood during postoperative adjuvant chemotherapy was related to recurrence or metastasis of NSCLC patients.

Keywords: Chemotherapy; epithelial cell adhesion molecule (EpCAM); circulating tumor cells (CTCs); nonsmall cell lung cancer (NSCLC); real-time fluorescence quantitative polymerase chain reaction (RTFQ-PCR)

Submitted Jan 31, 2021. Accepted for publication May 11, 2021.

doi: $10.21037 /$ tcr-21-205

View this article at: https://dx.doi.org/10.21037/tcr-21-205

\section{Introduction}

Lung cancer is the world's fastest growing morbidity and mortality rate, and it is the most malignant tumor that threatens human health and life $(1,2)$. Non-small cell lung cancer (NSCLC) accounts for about $85 \%$ of the incidence of lung cancer (3). With the continuous development 
of early diagnosis and treatment of lung cancer, more and more patients can undergo radical resection of lung cancer combined with postoperative adjuvant therapy (4). The current methods for monitoring the effectiveness of adjuvant therapy rely mainly on imaging (5). However, when imaging is diagnosed as recurrence or metastasis, it reaches stage $\mathrm{IV}$, and there are few effective treatments for stage IV patients $(6,7)$, and the 5 -year survival rate is even less than $2 \%(8,9)$. Therefore, there is an urgent need for an early indicator for monitoring the effect of adjuvant treatment after lung cancer surgery (10).

Since the discovery of circulating tumor cells (CTCs) and subsequent in-depth studies, it has been proven that the formation of CTCs precedes the formation of recurrence or metastases earlier than the imaging findings $(11,12)$. More studies have confirmed that the number of CTCs are related to the prognosis of NSCLC (13) and is an independent risk factor for prognosis (14). Epithelial cell adhesion molecule (EpCAM) can be as a specific marker for CTCs (15).

EpCAM is a multifunctional transmembrane glycoprotein expressed on both normal epithelium and epithelial neoplasms such as gastric, breast, and renal carcinomas (16). EpCAM is overexpressed in a wide variety of human cancers, including lung cancer, and its contribution to increased proliferation through upregulating cell cycle accelerators such as cyclin $\mathrm{A} / \mathrm{E}$ is well demonstrated in breast and gastric cancers (17). However, there are few reports about the effect of EpCAM on treatment and prognosis of NSCLC. In this study, the monitoring effects of EpCAM in CTCs on NSCLC patients with postoperative adjuvant treatment were explored. We present the following article in accordance with the STROBE reporting checklist (available at https:// dx.doi.org/10.21037/tcr-21-205).

\section{Methods}

\section{Patients}

A total of 76 patients were enrolled, aged between 36 and 70, who were treated in Department of Thoracic Surgery of our hospital from January 2017 to June 2018. Patients underwent radical resection of lung cancer and mediastinal lymph node dissection. They were diagnosed as primary NSCLC after operation with Ib-IIIa in terms of pTNM phases. They had never received any form of anti-tumor treatment before surgery and undergone adjuvant therapy based on NCCN Guidelines for NSCLC(2016 V4 version) (18).
The study was conducted in accordance with the Declaration of Helsinki (as revised in 2013). Patients had voluntarily signed informed consent and agreed to help with the research according to the experimental protocol with approval from Ethics Committee of Beijing Chest Hospital, Capital Medical University (NO.: YJS-2017-008).

\section{Sample collection}

At the 4th week after surgery, two drugs (platinumcontaining chemotherapeutics + platinum-free chemotherapeutics) first-line chemotherapy regimen were given according to the NCCN guidelines for NSCLC (2016 V4 version) (18), with 1 course every 21 days for a total of 4 courses. Patients with pN2 stage were given sequential radiotherapy. A total of $5 \mathrm{~mL}$ peripheral venous blood was extracted from median cubital vein with a blood collection tube containing K3-EDTA anticoagulant before surgery, on the seventh day after surgery, and one day before each adjuvant chemotherapy treatment, respectively. At each time that blood was extracted, the initial $3 \mathrm{~mL}$ blood was discarded, and then $2 \mathrm{~mL}$ of blood was obtained for testing, in order to avoid contamination of epithelial cells with skin tissue cells during puncture (a total of $5 \mathrm{~mL}$ blood extracted each time).

\section{Reverse transcript-polymerase chain reaction (RT-PCR)}

RT-PCR method was used to obtain EpCAM gene fragment from NSCLC cell line H2009 cells, and EpCAM was connected with pc-DNA3.1 vector to construct the target gene. The standardized recombinant plasmid was used as a positive standard for detection of real-time fluorescence quantitative polymerase chain reaction (RTFQ-PCR); sensitivity was verified by incorporating H2009 cells into peripheral blood; peripheral blood from patients with benign lung diseases and health volunteer were used as negative control to verify the specificity; cloned positive standards were diluted by $10^{7}-10^{1}$ copy gradients respectively to prepare a standard curve for RTFQ-PCR. The total RNA of mononuclear cells in whole blood was extracted from $2 \mathrm{~mL}$ of peripheral blood collected from patients, and cDNA was obtained by reverse transcription. The expression of EpCAM-mRNA was detected by RTFQ-PCR.

\section{Follow-up}

Chest CT, head CT and abdominal B-ultrasound were 
Table 1 The clinical features of patients

\begin{tabular}{|c|c|c|c|c|}
\hline Characteristic & Variables & Recurrence & Non-recurrence & $\mathrm{P}$ \\
\hline Gender & Female & 2 & 8 & \\
\hline \multirow[t]{2}{*}{ Age } & $<65$ & 13 & 39 & 0.230 \\
\hline & $\geq 65$ & 10 & 14 & \\
\hline Pathological patterns & $\begin{array}{l}\text { Squamous cell } \\
\text { carcinoma }\end{array}$ & 15 & 27 & \\
\hline \multirow[t]{2}{*}{ Clinical stages } & $\mathrm{lb}$ & 5 & 14 & 0.190 \\
\hline & Ila & 3 & 16 & \\
\hline
\end{tabular}

reviewed before the first and third chemotherapy for the patients who were enrolled in accordance with the NCCN guidelines for NSCLC (2016 V4 version) (18) during the postoperative adjuvant chemotherapy treatment. Patients had imaging review every 3 months within 2 years after the completion of adjuvant treatment. The end point of followup was imaging diagnosis of recurrence or metastasis. The diagnosis of NSCLC was to use CT to scan the brain, chest and abdomen to find that the lesion was a metastasis. Patients without recurrence or metastasis were followed up to 2 years after surgery. The recurrence was diagnosed vie CT scan on the brain, chest and abdomen for confirmation.

\section{Statistical analysis}

Statistical analysis was performed using SPSS 20.0. The experimental data were tested by Chi-square test. Analysis of covariance and post hoc test were used to compare EpCAM levels between two groups. All tests were performed using two-sided tests based on the actual clinical significance of the outcome. When $\mathrm{P}<0.05$, it was considered statistically significant.

\section{Results}

\section{Basic information of the enrolled patients}

This study enrolled 76 patients, including 66 male patients and 10 female patients; age $58.63 \pm 8.27$ years (minimum 36 years old, maximum 70 years old); pathological type:
34 cases of adenocarcinoma, 42 cases of squamous cell carcinoma; clinical stage: 19 cases of stage Ib, 19 cases of stage IIa, 9 cases of stage IIb, and 29 cases of stage IIIa. Recurrence was found in 23 cases, including 21 men and 2 women; 13 cases $<65$ years and 10 cases $\geq 65$ years; 8 cases of adenocarcinoma and 15 cases of squamous cell carcinoma; 5 cases of stage Ib, 3 cases of stage IIa, 2 cases of stage IIb, 13 cases of stage IIIa. The cases included in this study were staged from Ib to IIIa, there were no locally advanced cases, and all cases reached $\mathrm{R} 0$ resection. There were 53 cases with no recurrence, including 45 men and 8 women; 39 cases $<65$ years and 14 cases $\geq 65$ years; 26 cases of adenocarcinoma and 27 cases of squamous cell carcinoma; 14 cases of stage Ib, 16 cases of stage IIa, and 7 cases of stage IIb 16 cases of stage IIIa (Table 1).

\section{Monitoring results of EpCAM expression in peripheral blood CTCs during postoperative adjuvant therapy}

EpCAM expression level of CTCs before surgery was $315.82 \pm 289.83$ copies $/ 2 \mathrm{~mL}$. EpCAM expression level of peripheral blood CTCs before operation was $179.17 \pm 193.33$ copies $/ 2 \mathrm{~mL}$. The expression levels of EpCAM in CTCs between the recurrent group and the non-recurrent group at 1 day before surgery, first, second and third adjuvant chemotherapy showed no significant difference $(\mathrm{P}>0.05)$. Only one day before the fourth adjuvant chemotherapy treatment, there was a statistically significant difference in EpCAM expression levels in peripheral blood CTCs between the recurrent group and 
Table 2 Comparison of the expression levels of EpCAM of CTCs between the recurrent group and non-recurrent group

\begin{tabular}{lccc}
\hline Time & Recurrent group & Non-recurrent group & $P$ \\
\hline Before surgery & $315.82 \pm 289.83$ & $179.17 \pm 193.33$ & $44.92 \pm 29.66$ \\
After surgery & $49.47 \pm 38.21$ & $44.85 \pm 20.72$ & 0.588 \\
Before the first chemotherapy & $52.37 \pm 18.03$ & $45.09 \pm 23.11$ & 0.203 \\
Before the second chemotherapy & $50.54 \pm 23.94$ & $40.44 \pm 22.38$ & 0.418 \\
Before the third chemotherapy & $52.60 \pm 29.97$ & $36.25 \pm 17.90$ & 0.008
\end{tabular}

Data were expressed as mean \pm SD.

the non-recurrent group $(\mathrm{P}=0.008)$ (Table 2).

\section{Follow-up results}

As of January 2019, the average follow-up time of 76 patients was $13.12 \pm 5.32$ months, and 23 patients had recurrence or metastasis at the endpoint of follow-up. The mean follow-up time for imaging diagnosis of recurrence or metastasis in 23 patients was $8.00 \pm 4.91$ months, and the monitoring of EpCAM expression in peripheral blood CTCs was 3.25 months on average. There was a significant difference between the time of imaging diagnosis of recurrence or metastasis and the time of monitoring the expression levels of EpCAM in peripheral blood CTCs $(\mathrm{P}<0.0001)$.

\section{Discussion}

EpCAM is a Type I transmembrane superficial glycoprotein antigen that is expressed on the surface of basolateral membrane of multiple epithelial cells with some exceptions (19). Previous study demonstrated that EpCAM substantially contributed to the pathogenesis of lung cancer, especially in its survival, and the development of EpCAM-targeted therapy for lung cancer may have promise (17). In this study, RTFQ-PCR was used to detect EpCAM expression levels of 76 cases with NSCLC received complete postoperative adjuvant chemotherapy. The recurrence or metastasis diagnosed by imaging were used as the end point to compare EpCAM expression levels in CTCs during the adjuvant chemotherapy treatment between the recurrence and non-recurrence groups. The relationship between EpCAM expression levels in CTCs and the effect of adjuvant chemotherapy on patients with NSCLC after surgery was initially explored.
After the above-mentioned 76 patients were followed up, there were 23 cases of recurrence or metastasis and 53 cases without recurrence or metastasis, confirmed by imaging. There were no significant differences between the recurrence group and the non-recurrence group in gender, age, pathological type of NSCLC, clinical stage, and EpCAM expression levels of CTCs in preoperative peripheral blood $(\mathrm{P}>0.05)$.

Although the EpCAM expression levels were monitored at 5 time points during postoperative adjuvant chemotherapy treatment, there was only a statistical difference in EpCAM expression level of peripheral blood CTCs on 1 day before the 4th adjuvant chemotherapy. In the recurrent group, the expression level of EpCAM at first day before the first adjuvant chemotherapy was higher than that in the first day after the second adjuvant chemotherapy, suggesting that adjuvant therapy was effective. The expression level of EpCAM continued to increase from 1 day before the third adjuvant chemotherapy, suggesting that adjuvant treatment was ineffective. In the non-recurrent group, EpCAM expression level in cells was basically stable from 1 day before the second adjuvant chemotherapy treatment. EpCAM expression level continued to decrease from the day before the third adjuvant chemotherapy, suggesting that adjuvant treatment was effective.

Comparison of recurrent group and non-recurrent group, there were no significant differences in EpCAM expression levels on the 7 th day after surgery and the 1 st day before the 1st, $2 \mathrm{nd}$, and 3 rd adjuvant chemotherapy treatments $(\mathrm{P}>0.05)$. EpCAM expression levels in peripheral blood CTCs 1 day before the 4th adjuvant chemotherapy treatment were statistically different between the recurrent and non-recurrent groups $(\mathrm{P}=0.008)$, indicating that EpCAM expression levels could reflect the effect of adjuvant chemotherapy. 
The average time for imaging diagnosis of recurrence or metastasis was $8.00 \pm 4.91$ months (minimum 2 months, maximum 21 months). The average monitoring time for the first day before the 4th adjuvant chemotherapy was 3.25 months. The statistical analysis of the time of recurrence or metastasis diagnosed by imaging analysis was significantly different from the monitoring time of EpCAM expression at 1 day before the 4th adjuvant chemotherapy treatment $(\mathrm{P}<0.0001)$, indicating that time for statistically significant changes in EpCAM expression levels of recurrence group and non-recurrent group to diagnoses recurrence or metastasis was earlier than imaging. In previous study, known clinical variables including age of patient, gender, histology, stage of disease, and response to chemotherapy were not found to be correlated with serum EPCAM concentrations $(\mathrm{P}>0.05)$, indicating that serum EpCAM concentrations had no diagnostic, predictive, and prognostic roles in patients with lung cancer (20).

The content of CTCs in the blood of patients with NSCLC is extremely rare. About $10^{5-7}$ monocytes contain only one CTC, so it was difficult to detect CTCs. Enrichment of CTCs in blood samples before detection can increase the detection rate, but the enrichment method not only increases the detection cost, but the enrichment step can also cause the loss of CTCs. In this study, the fluorescence quantitative PCR method was highly sensitive. The incorporation test of NSCLC was carried out when the method was established, and the detection requirements can be met without enrichment. Therefore, CTC enrichment was not performed before RNA extraction. The advantage of this detection method was that it had high sensitivity and low cost. It can detect changes in EpCAM expression of CTCs in blood samples as early as possible after surgery. The disadvantage was that this detection method was to extract RNA after destroying CTCs. If the cell morphology was destroyed, false positives cannot be eliminated by morphology. To make up for this, both benign lung diseases and healthy volunteers were tested when the experimental method was established. The EpCAM expression level of CTCs in the peripheral blood of 200 patients with NSCLC before surgery was $217.50 \pm 244.07$ copies $/ 2 \mathrm{~mL}$. The EpCAM expression level of CTCs in the peripheral blood of 50 patients with benign lung diseases before operation was $3.93 \pm 2.70$ copies $/ 2 \mathrm{~mL}$. The EpCAM expression levels of CTCs in the peripheral blood of 400 healthy volunteers was $3.56 \pm 2.05$ copies $/ 2 \mathrm{~mL}$.

EpCAM expression levels of peripheral blood CTCs were $315.82 \pm 289.83$ copies $/ 2 \mathrm{~mL}$ in recurrent group and $179.17 \pm 193.33$ copies $/ 2 \mathrm{~mL}$ in the non-recurrent group, and the comparison of the two groups showed no statistical difference $(\mathrm{P}=0.084)$. This is inconsistent with the conclusions obtained by most studies that CTCs are independent prognostic risk factors (21-23). The level of EpCAM expression in peripheral blood CTCs before and after the first, second, and third adjuvant chemotherapy treatments did not show statistical differences, which may be related to the small sample size and insufficient followup time. More cases need to be included and continue to complete or extend the follow-up time.

From the individual perspective, there was an inverse overall trend change. For example, in the No. 3 patient in recurrent group, EpCAM expression levels in peripheral blood CTCs monitored at 5 time points after stage IIa adenocarcinoma were stable (4.56 copies $/ 2 \mathrm{~mL}$ ), 5.51 copies $/ 2 \mathrm{~mL}, 5.01$ copies $/ 2 \mathrm{~mL}, 4.89$ copies $/ 2 \mathrm{~mL}$, and 5.32 copies/2 mL (Figure 1), but No. 3 patient was diagnosed with a lumbar 4-vertebral body metastasis at 6 months after surgery. This phenomenon may be related to epithelial-mesenchymal transition in CTCs (24). Due to internal or external factors, the specific marker of epithelial cell in the tumor cells, EpCAM, which has phenotypic changes, might show down-regulated expression or expression loss, while interstitial specific marker shows upregulated expression, which is the epithelial-mesenchymal transition (25). Therefore, the detection method that depends on EpCAM as a specific marker in tumor cells will miss out those circulating cells that show a downregulated expression or expression loss of EpCAM (26). This requires the combination of other interstitial markers or the identification of specific tumor cell interstitial markers, but to date no highly specific tumor interstitial markers have been identified (10). Due to the lack of interstitial-specific markers, the method of detecting CTCs by virtue of epithelialspecific markers is still commonly used in current research and clinical testing, but the method for detecting interstitial CTCs is still needed. Although there is no statistical difference in the expression levels of EpCAM cells between the recurrent group and the non-recurrent group at 1 day before the first adjuvant chemotherapy, the trend in Table 2 showed that the increase of EpCAM expression in peripheral blood circulation at this time indicated a poor prognosis, which could guide the formulation of postoperative adjuvant chemotherapy regimens. In addition, although there was a statistical difference in the expression levels of EpCAM between the recurrent group and the non-recurrent group only at 1 day before the 4th adjuvant chemotherapy, 


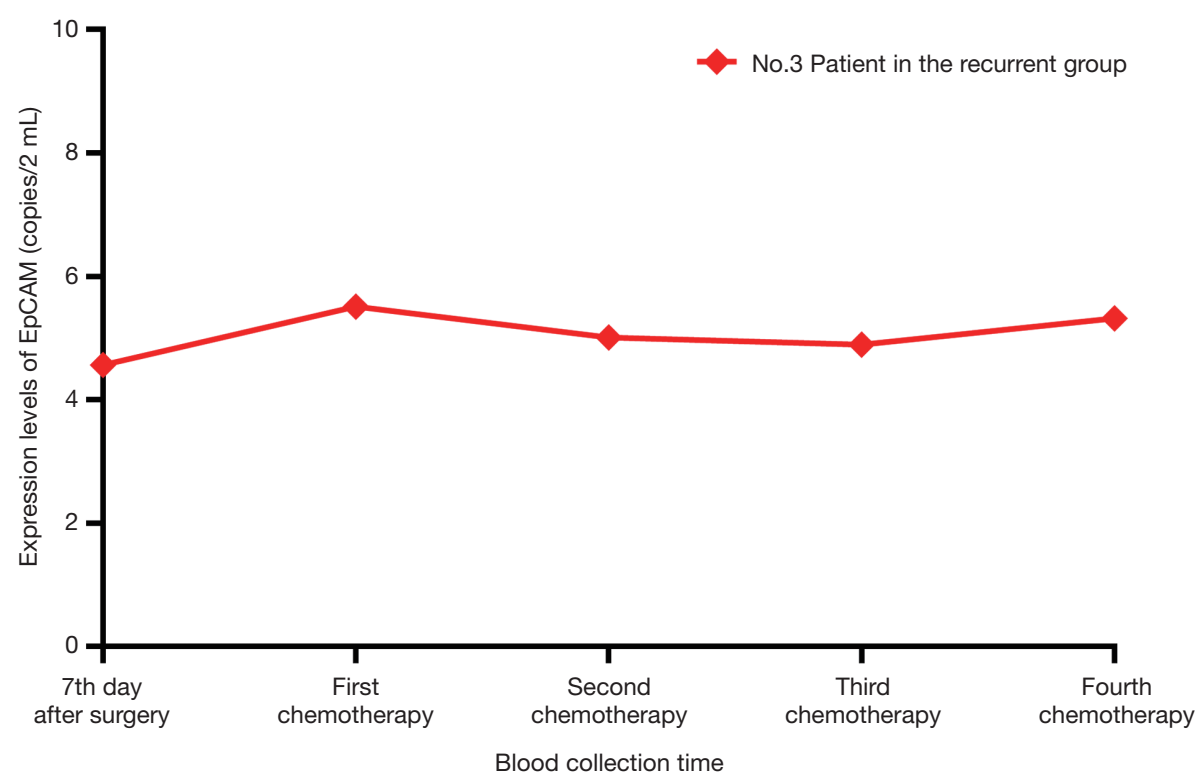

Figure 1 The expression levels of EpCAM of CTCs in the No. 3 Patient in the recurrent group at the 7th day after surgery, the first chemotherapy, the second chemotherapy, the third chemotherapy, and the fourth chemotherapy.

it could be seen from Table 2 that the inflection point appears after 1 day before the 2 nd adjuvant chemotherapy, suggesting that the detection of EpCAM expression levels after the second chemotherapy may reflect the effect of adjuvant chemotherapy, and thus guide the adjustment of individualized adjuvant chemotherapy schemes early.

\section{Conclusions}

In conclusion, our results demonstrated that EpCAM expression levels of CTCs in NSCLC patients during postoperative adjuvant chemotherapy were related to recurrence or metastasis, and were earlier than those diagnosed by CT imaging. However, due to small sample size, the further study in large population is still needed.

\section{Acknowledgments}

Funding: None.

\section{Footnote}

Reporting Checklist: The authors have completed the STROBE reporting checklist. Available at https://dx.doi. org/10.21037/tcr-21-205

Data Sharing Statement: Available at https://dx.doi. org/10.21037/tcr-21-205

Conflicts of Interest: All authors have completed the ICMJE uniform disclosure form (available at https://dx.doi. org/10.21037/tcr-21-205). The authors have no conflicts of interest to declare.

Ethical Statement: The authors are accountable for all aspects of the work in ensuring that questions related to the accuracy or integrity of any part of the work are appropriately investigated and resolved. The study was conducted in accordance with the Declaration of Helsinki (as revised in 2013). The study was approved by institutional ethics board of Beijing Chest Hospital, Capital Medical University (NO.: YJS-2017-008) and informed consent was taken from all the patients.

Open Access Statement: This is an Open Access article distributed in accordance with the Creative Commons Attribution-NonCommercial-NoDerivs 4.0 International License (CC BY-NC-ND 4.0), which permits the noncommercial replication and distribution of the article with the strict proviso that no changes or edits are made and the original work is properly cited (including links to both the formal publication through the relevant DOI and the license). See: https://creativecommons.org/licenses/by-nc$\mathrm{nd} / 4.0 /$. 


\section{References}

1. Siegel RL, Miller KD, Jemal A. Cancer statistics, 2019. CA Cancer J Clin 2019;69:7-34.

2. Chen $W$, Zheng R, Baade PD, et al. Cancer statistics in China, 2015. CA Cancer J Clin 2016;66:115-32.

3. Majem M, Juan O, Insa A, et al. SEOM clinical guidelines for the treatment of non-small cell lung cancer (2018). Clin Transl Oncol 2019;21:3-17.

4. National Lung Screening Trial Research Team; Aberle DR, Adams AM, et al. Reduced lung-cancer mortality with low-dose computed tomographic screening. N Engl J Med 2011;365:395-409.

5. Hirsch FR, Scagliotti GV, Mulshine JL, et al. Lung cancer: current therapies and new targeted treatments. Lancet 2017;389:299-311.

6. Henschke CI, Yip R, Yankelevitz DF, et al. Definition of a positive test result in computed tomography screening for lung cancer: a cohort study. Ann Intern Med 2013;158:246-52.

7. Francis S, Orton A, Stoddard G, et al. Sequencing of Postoperative Radiotherapy and Chemotherapy for Locally Advanced or Incompletely Resected Non-SmallCell Lung Cancer. J Clin Oncol 2018;36:333-41.

8. Horeweg N, van Rosmalen J, Heuvelmans MA, et al. Lung cancer probability in patients with CT-detected pulmonary nodules: a prespecified analysis of data from the NELSON trial of low-dose CT screening. Lancet Oncol 2014;15:1332-41.

9. Ferlay J, Soerjomataram I, Dikshit R, et al. Cancer incidence and mortality worldwide: sources, methods and major patterns in GLOBOCAN 2012. Int J Cancer 2015;136:E359-86.

10. Vargas AJ, Harris CC. Biomarker development in the precision medicine era: lung cancer as a case study. Nat Rev Cancer 2016;16:525-37.

11. Huang MY, Tsai HL, Huang JJ, et al. Clinical Implications and Future Perspectives of Circulating Tumor Cells and Biomarkers in Clinical Outcomes of Colorectal Cancer. Transl Oncol 2016;9:340-7.

12. Beck TN, Boumber YA, Aggarwal C, et al. Circulating tumor cell and cell-free RNA capture and expression analysis identify platelet-associated genes in metastatic lung cancer. BMC Cancer 2019;19:603.

13. Murlidhar V, Reddy RM, Fouladdel S, et al. Poor Prognosis Indicated by Venous Circulating Tumor Cell Clusters in Early-Stage Lung Cancers. Cancer Res 2017;77:5194-206.

14. de Bono JS, Scher HI, Montgomery RB, et al. Circulating tumor cells predict survival benefit from treatment in metastatic castration-resistant prostate cancer. Clin Cancer Res 2008;14:6302-9.
15. Skirecki T, Hoser G, Kawiak J, et al. Flow cytometric analysis of CD133- and EpCAM-positive cells in the peripheral blood of patients with lung cancer. Arch Immunol Ther Exp (Warsz) 2014;62:67-75.

16. Wang A, Ramjeesingh $\mathrm{R}$, Chen $\mathrm{CH}$, et al. Reduction in membranous immunohistochemical staining for the intracellular domain of epithelial cell adhesion molecule correlates with poor patient outcome in primary colorectal adenocarcinoma. Curr Oncol 2016;23:e171-8.

17. Hase T, Sato M, Yoshida K, et al. Abstract 1657: A pivotal role for epithelial cell adhesion molecule (EpCAM) in survival of lung cancer cells. Cancer Res 2011;71:1657.

18. Ettinger DS, Wood DE, Akerley W, et al. NCCN Guidelines Insights: Non-Small Cell Lung Cancer, Version 4.2016. J Natl Compr Canc Netw 2016;14:255-64.

19. Yahyazadeh Mashhadi SM, Kazemimanesh M, Arashkia A, et al. Shedding light on the EpCAM: An overview. J Cell Physiol 2019;234:12569-80.

20. Tas F, Karabulut S, Duranyildiz D. Clinical significance of serum epithelial cell adhesion molecule (EPCAM) levels in patients with lung cancer. Mol Cell Biochem 2014;396:307-12.

21. Pirro M, Cagini L, Paciullo F, et al. Baseline and post-surgery endothelial progenitor cell levels in patients with early-stage non-small-cell lung carcinoma: impact on cancer recurrence and survival. Eur J Cardiothorac Surg 2013;44:e245-52.

22. O'Flaherty JD, Gray S, Richard D, et al. Circulating tumour cells, their role in metastasis and their clinical utility in lung cancer. Lung Cancer 2012;76:19-25.

23. Kuwata T, Yoneda K, Kobayashi K, et al. Circulating Tumor Cells as an Indicator of Postoperative Lung Cancer: A Case Report. Am J Case Rep 2016;17:663-5.

24. Rhim AD, Mirek ET, Aiello NM, et al. EMT and dissemination precede pancreatic tumor formation. Cell 2012;148:349-61.

25. Chaffer CL, Weinberg RA. A perspective on cancer cell metastasis. Science 2011;331:1559-64.

26. Austin RG, Huang TJ, Wu M, et al. Clinical utility of non-EpCAM based circulating tumor cell assays. Adv Drug Deliv Rev 2018;125:132-42.

Cite this article as: Zhen DZ, Li YS, Su CY, Cheng X, Zhou SJ, Han Y, Yu DP, Song XY, Xiao N, Liu ZD, Wang F. Expression level of epithelial cell adhesion molecule (EpCAM) of circulating tumor cells (CTCs) of patients with NSCLC as an early indicator to monitor the effects of postoperative adjuvant chemotherapy. Transl Cancer Res 2021;10(7):32993305. doi: 10.21037/tcr-21-205 